\title{
Efecto de la deficiencia subclínica de zinc en corderos sobre retención de nitrógeno, parámetros productivos y hematológicos
}

\section{Effect of subclinical zinc deficiency in lambs on nitrogen retention, productive and hematological parameters}

\section{Efeito da deficiência subclínica de zinco em cordeiros na retenção de nitrogênio, parâmetros produtivos e hematológicos}

\author{
Pechin $\mathrm{GH}^{1}$, Corbellini $\mathrm{CN}^{2}$, Denda $S S^{1}$ \\ 1 Facultad de Ciencias Veterinarias, UNLPam, calle 5 esq. 116 (6360) General Pico, La Pampa. \\ 2 INTA Mercedes, Buenos Aires (retirado).
}

Correo electrónico: ghpechin@vet.unlpam.edu.ar

\section{RESUMEN}

El objetivo de este ensayo fue estudiar el efecto de la deficiencia subclínica de zinc ( $\mathrm{Zn}$ ) en corderos sobre variables de interés productivo, el balance de nitrógeno (N) y parámetros hematológicos. Diez corderos de raza Corriedale, con un peso promedio de $10,09 \mathrm{~kg}$, fueron asignados aleatoriamente a dos grupos: Basal (B, 10 ppm de Zn) y Suplementado con 30 ppm de Zn (Z). El ensayo se extendió por el término de 20 semanas y los animales fueron pesados cada 4 semanas. También con esta periodicidad, se obtuvieron muestras de sangre por punción de vena yugular. En las semanas 6 y 20 se evaluó la digestibilidad aparente de la materia seca (DAMS) y el balance de $\mathrm{N}$. Al final del ensayo se midió la producción y la calidad de la lana de los corderos. La ganancia de peso y la calidad de la lana fueron similares en ambos grupos, mientras que la producción de lana fue un 37,4 \% superior en el grupo Z. De acuerdo al primer periodo de balance, la DAMS fue similar en ambos grupos, pero la retención de $\mathrm{N}$ fue significativamente 
mayor en el grupo $\mathrm{Z}$ que en el grupo B (5,53 vs 4,03 g/día). En el segundo periodo de balance hubo una tendencia similar. No hubo diferencias entre los grupos en hematocrito, concentración de hemoglobina, conteo de glóbulos rojos, leucocitos totales, linfocitos y neutrófilos. Estos resultados sugieren que la deficiencia subclínica de $\mathrm{Zn}$ reduce la producción de lana, lo que puede estar relacionado con una menor síntesis proteica.

Palabras clave: deficiencia de Zn, corderos, producción de lana, retención de nitrógeno, hematología.

\section{ABSTRACT}

The objective of this trial was to study the effect of subclinical zinc (Zn) deficiency in lambs on productive variables, nitrogen $(\mathrm{N})$ balance and hematological parameters. Ten Corriedale lambs, with an average weight of $10.09 \mathrm{~kg}$, were randomly assigned to two groups: Basal (B, $10 \mathrm{ppm} \mathrm{Zn)} \mathrm{and} \mathrm{Supplemented} \mathrm{with} 30 \mathrm{ppm} \mathrm{Zn} \mathrm{(Z).} \mathrm{The} \mathrm{trial} \mathrm{lasted} 20$ weeks. The animals were weighed every 4 weeks. Also with this periodicity, they were bled by jugular venipuncture. In weeks 6 and 20 the apparent dry matter digestibility (ADMD) and $\mathrm{N}$ balance were evaluated. At the end of the test the wool production and quality were measured. The weight gain and wool quality were similar in both groups, while wool production was $37.4 \%$ higher in group Z. According to the first balance period, ADMD was similar in both groups, but the retention of $\mathrm{N}$ was significantly higher in group $\mathrm{Z}$ than in group B (5.53 vs $4.03 \mathrm{~g} /$ day). In the second balance period there was a similar trend. There were no differencies between groups on hematocrit, hemoglobin concentration, red blood cells, total leukocytes, lymphocytes and neutrophils number. These results suggest that subclinical $\mathrm{Zn}$ deficiency reduces wool production, which may be related to lower protein synthesis.

Key words: zinc deficiency, lambs, wool production, nitrogen retention, hematology.

\section{RESUMO}

O objetivo deste teste foi estudar o efeito da deficiência subclínica de zinco (Zn) em cordeiros sobre variáveis de interesse produtivo, o balanço de nitrogênio (N) e parâmetros hematológicos. Dez cordeiros da raça Corriedale, com peso médio de 10,09 kg, foram 
divididos aleatoriamente em dois grupos: Basal (B, 10 ppm de Zn) e Suplementado com 30 ppm de Zn (Z). 0 teste estendeu-se durante 20 semanas e os animais foram pesados a cada 4 semanas. Também com essa frequência, foram obtidas amostras de sangue por punção da veia jugular. Nas semanas 6 e 20, foram avaliadas a digestibilidade aparente da matéria seca (DAMS) e o balanço de N. Ao final do teste, foram medidas a produção e a qualidade da lã dos cordeiros. 0 ganho de peso e a qualidade da lã foram semelhantes em ambos os grupos, enquanto a produção de lã foi 37,4\% superior no grupo Z. De acordo com o primeiro período de balanço, a DAMS foi semelhante nos dois grupos, mas a retenção de $\mathrm{N}$ foi significativamente maior no grupo $\mathrm{Z}$ do que no grupo B (5,53 vs 4,03 g /dia). No segundo período do balanço houve uma tendência semelhante. Não houve diferenças entre os grupos no hematócrito, concentração de hemoglobina, contagem de glóbulos vermelhos, leucócitos totais, linfócitos e neutrófilos. Estes resultados sugerem que a deficiência subclínica de Zn reduz a produção de lã, o que pode estar relacionado com uma menor síntese protéica.

Palavras-chave: deficiência de $\mathrm{Zn}$, cordeiros, produção de lã, retenção de nitrogênio, hematologia

Fecha de recepción artículo original: 05-02-2021

Fecha de aceptación para su publicación: 15-03-2021

\section{Introducción}

La deficiencia experimental de zinc ( $\mathrm{Zn}$ ) fue descripta por primera vez en ovinos por Ott et al. ${ }^{(1)} \mathrm{A}$ partir de entonces, se han sucedido varios trabajos que profundizaron en el tema. También existen informes de casos de deficiencia clínica de Zn ocurridos naturalmente en esta especie ${ }^{(2,3,4,5)}$.

La deficiencia de $\mathrm{Zn}$ en corderos ( $5 \mathrm{ppm}$ de $\mathrm{Zn}$ en la dieta, base materia seca, MS) afecta severamente la ganancia de peso, mientras que produce el cese de la producción de lana en el término de 2 semanas de depleción ${ }^{6}$. Similares resultados fueron alcanzados con corderos alimentados con dietas semisintéticas que contenían 2,7 a 4 ppm de $\mathrm{Zn}^{(1,7,8,9)}$.

La disminución del crecimiento causada por la deficiencia de $\mathrm{Zn}$ es debida, fundamentalmente, a un menor consumo de alimento, aunque se observó que la ganancia de peso era aún menor en los animales deficientes que en el grupo pareado por consumo, lo que indica una menor eficiencia de conversión alimenticia ${ }^{(10,11)}$, ya que la digestibilidad aparente de la MS (DAMS) no parece ser afectada por la deficiencia de 
$\mathrm{Zn}^{(12)}$. En relación a este problema, se halló también una menor retención de nitrógeno $(\mathrm{N})$ en los corderos deficientes en comparación con los controles pareados por consumo, causada por una similar digestibilidad de la proteína, pero una mayor excreción urinaria de $\mathrm{N}^{(11)}$.

Los resultados de McNall et al. en ratas ${ }^{(13)}$ sugieren que la menor ganancia de peso está también relacionada con una disminución de los receptores para la hormona de crecimiento (GH) en el hígado y de la síntesis del factor de crecimiento I similar a la insulina (IGF-I). Lo mismo parece suceder en otras especies. Droke et al. ${ }^{(14)}$ hallaron, en corderos deficientes en $\mathrm{Zn}$, niveles similares de GH en respuesta a la hormona liberadora de somatotrofina, pero menores niveles de IGF-I que en el grupo control. Además de ello, se sugieren efectos de la deficiencia de Zn sobre la proliferación celular, que se producen a nivel de la señalización intracelular en respuesta a IGF-I ${ }^{(15)}$.

La deficiencia de Zn, en humanos y animales, causa atrofia de timo, linfopenia y una menor respuesta de la inmunidad celular y humoral. A medida que la depleción progresa, ocurre una reprogramación del sistema inmunitario, con disminución en la linfopoyesis y preservación de la mielopoyesis ${ }^{(16)}$. En línea con ello, Droke y Spears ${ }^{(8)}$ han hallado, en corderos deficientes en Zn, una disminución de linfocitos circulantes y un incremento de neutrófilos.

La mayoría de los trabajos realizados en animales incluyeron modelos que producen una deficiencia clínica de Zn. Por ello, resulta de interés profundizar en el estudio de los parámetros más sensibles que puedan colaborar en el diagnóstico de una deficiencia moderada o subclínica del mineral y sus efectos sobre las variables productivas, siguiendo un modelo que produce una disminución significativa del $\mathrm{Zn}$ sérico, de la concentración de $\mathrm{Zn}$ en hueso y de la retención de $\mathrm{Zn}^{(17)}$.

Los objetivos de este ensayo incluyeron verificar el efecto de la deficiencia subclínica de $\mathrm{Zn}$ en corderos sobre ganancia de peso, producción y calidad de lana, DAMS, retención de $\mathrm{N}$ y parámetros hematológicos.

\section{Materiales y métodos}

Diez corderos de raza Corriedale, con un peso de 10,09 $\pm 1,285 \mathrm{~kg}$, fueron asignados en forma aleatoria, estratificando por peso inicial, a dos grupos: Basal (B) y Suplementado con Zn (Z). Ambos grupos recibieron una dieta detallada en la Tabla 1. Esta dieta fue similar a la utilizada en su ensayo por White et al. ${ }^{(9)}$, con algunas modificaciones: se disminuyó el contenido de paja de trigo, se incrementó el de albúmina de huevo y se suplantó el aceite de soja por el de girasol. 
Tabla 1: Dieta experimental (composición porcentual, base alimento como tal).

\begin{tabular}{|c|c|}
\hline Ingrediente & Porcentaje \\
\hline Albúmina de huevo deshidratada & 10 \\
\hline Urea & 2 \\
\hline Almidón de maíz & 34,8 \\
\hline Sacarosa & 14 \\
\hline Paja de trigo & 30 \\
\hline Núcleo mineral-vitamínico sin $\mathrm{Zn}$ & 4,2 \\
\hline Bicarbonato de sodio & 3 \\
\hline Aceite de girasol & 2 \\
\hline
\end{tabular}

Dicha dieta fue preparada en el laboratorio, y se transportó hasta el galpón en recipientes plásticos tapados. El mezclado de la dieta se facilitó al moler la paja, lográndose fragmentos entre 0,5 y $2 \mathrm{~cm}$ de largo de la misma, los que cumplen los requerimientos mínimos para la "fibra efectiva" (tamaño de partícula que estimula la rumia y la salivación).

El alimento se administró dos veces al día, por la mañana y por la tarde, en forma restringida, a un nivel que fluctuó entre el 2,7 y el $3 \%$ del peso de los animales. La dieta B contenía 10 ppm de Zn, base MS, y la dieta $\mathrm{Z}$ fue suplementada con $\mathrm{ZnSO}_{4} \cdot \mathrm{H}_{2} \mathrm{O}$ (Sulfato de zinc), para aportar un nivel extra de $30 \mathrm{ppm}$ de $\mathrm{Zn}$ y cumplir las recomendaciones del NRC ${ }^{(18)}$.

El protocolo de manejo de los animales fue aprobado por el Comité de Ética de la Facultad de Ciencias Veterinarias, UNLPam. Los corderos fueron alojados en jaulas de plástico, de $1 \mathrm{~m}$ x 0,5 m, con piso ranurado. Se procedió con un período de destete y acostumbramiento de dos semanas, durante las cuales los animales fueron alimentados con gramíneas (cebadilla criolla, Bromus unioloides, en su mayor parte), cortadas y ofrecidas en estado fresco. El ensayo tuvo una duración de 20 semanas. Al inicio del ensayo, se esquiló un área de $80 \mathrm{~cm}^{2}$, del flanco de cada cordero, con el objeto de medir el crecimiento y la calidad de la lana en ambos grupos. Los animales se pesaron cada 4 semanas, para evaluar la evolución de la ganancia de peso. Con la misma periodicidad, pero a partir de la octava semana, fueron sangrados de vena yugular y se extrajo una muestra de sangre con EDTA sódico como anticoagulante, para realizar el conteo de glóbulos rojos, leucocitos y linfocitos, la medición del hematocrito y la determinación de la concentración de hemoglobina. En la sexta y en la última semana del trabajo se evaluaron la DAMS y el balance de N. Durante cada período 
de balance ( 5 días) se midió, en forma diaria, el alimento consumido por cada animal y la producción de heces y de orina. Se tomó una alícuota diaria de cada excreta ( 30 gr para heces y $20 \mathrm{ml}$ para orina), y se guardaron a - $20^{\circ} \mathrm{C}$ hasta su análisis. A partir de estos datos, se calculó la cantidad de $\mathrm{N}$ retenido por cada animal.

Al finalizar el ensayo, se cortó la lana de las áreas previamente delimitadas y se pesó para medir la producción de lana durante el período. Una muestra de cada animal fue procesada para determinar los parámetros de calidad en el Laboratorio de Calidad de Lanas de INTA Bariloche: diámetro de lana (analizador Sirolan-Laserscan, método IWTO-12-00) ${ }^{(19)}$, curvatura de lana (analizador Sirolan-Laserscan) ${ }^{(20)}$ y resistencia a la tracción (método IWT0-30-98) ${ }^{(21)}$.

Las muestras de alimento y heces fueron secadas a $100{ }^{\circ} \mathrm{C}$ para la determinación de MS y a $60{ }^{\circ} \mathrm{C}$ para la medición de $\mathrm{N}$, y luego fueron molidas en un molino a martillo tipo Resch. El $\mathrm{N}$ total de alimento, heces y orina fue determinado por la técnica semi-micro Kjeldahl ${ }^{(22)}$.

Análisis estadístico: Los datos fueron analizados en un diseño completamente aleatorio. Para las variables hematocrito, concentración de hemoglobina en sangre y número de glóbulos rojos, leucocitos y linfocitos en sangre se utilizó un modelo mixto con mediciones repetidas en el tiempo (PROC MIXED, SAS/STAT 9.1) ${ }^{(23,24)}$. El modelo incluyó los efectos fijos de tratamiento, tiempo e interacción tratamiento $\mathrm{x}$ tiempo, $\mathrm{y}$ el efecto aleatorio del animal dentro de tratamiento. Se usó una estructura de covarianza autoregresiva de orden 1 para varianzas homogéneas (AR1). Para el resto de las variables (ganancia de peso, producción y calidad de lana, DAMS y retención de N) se utilizó el procedimiento PROC GLM, del mismo paquete estadístico. Los efectos fueron considerados significativos cuando $\mathrm{P}<0,05$ y tendencias cuando $\mathrm{P}<0,10$.

\section{Resultados}

\section{A. GANANCIA DE PESO, PRODUCCIÓN Y CALIDAD DE LANA.}

En ningún momento del ensayo los animales mostraron signos clínicos de deficiencia de $\mathrm{Zn}$. La ganancia de peso de los dos grupos de corderos fue similar (Tabla 2 y Figura 1), y estuvo influida por episodios de acidosis, con la consecuente disminución del consumo, que afectaron indistintamente a miembros de cualquiera de los dos grupos. La inclusión de $3 \%$ de bicarbonato de sodio a la ración contribuyó al control de este problema sólo en forma parcial. 
La producción de lana sucia fue un 37,4 \% más alta en el grupo suplementado con Zn (Tabla 2). Sin embargo, no hubo diferencia en ninguno de los parámetros de calidad de lana evaluados (Tabla 3).

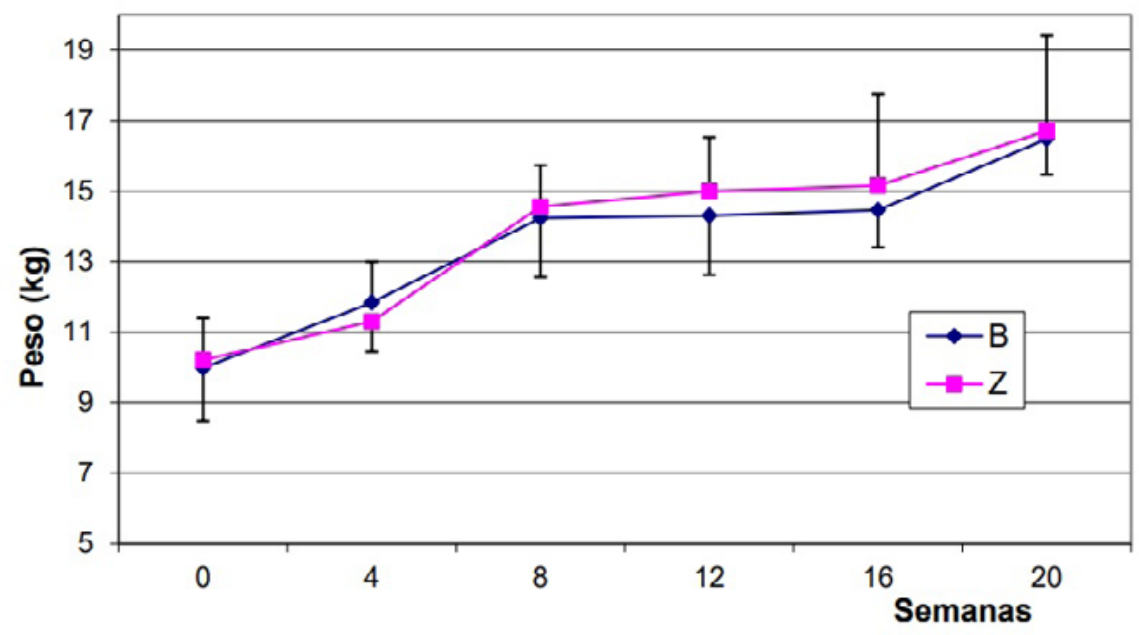

Figura 1: Evolución del peso de los corderos de ambos grupos experimentales

Tabla 2: Ganancia de peso y producción de lana sucia en ambos grupos (valores expresados como promedio $\pm \mathrm{DE})$.

${ }^{x}$ en un área de $80 \mathrm{~cm}^{2}$.

\begin{tabular}{|c|c|c|c|}
\hline Variable & Grupo B & Grupo Z & P \\
\hline Ganancia de peso $(\mathrm{kg})$ & $6,5 \pm 1,23$ & $6,5 \pm 1,90$ & 0,99 \\
\hline${\text { Producción de lana sucia }(\mathrm{g})^{\mathrm{x}}}$ & $15,94 \pm 2,42$ & $21,90 \pm 1,46$ & $<0,01$ \\
\hline
\end{tabular}

Tabla 3: Calidad de la lana de los corderos de ambos grupos experimentales (valores expresados como promedio $\pm \mathrm{DE}$ ).

\begin{tabular}{|c|c|c|c|}
\hline Variable & Grupo B & Grupo Z & P \\
\hline Diámetro de fibras $(\mu)$ & $22,66 \pm 1,74$ & $21,74 \pm 1,59$ & 0,4090 \\
\hline Curvatura de ondulación $($ grados $/ \mathrm{mm})$ & $76,49 \pm 8,36$ & $79,62+6,79$ & 0,5343 \\
\hline Fibras con diámetro $>30 \mu(\%)$ & $9,78 \pm 4,78$ & $8,96 \pm 4,57$ & 0,7886 \\
\hline Resistencia a la tracción $($ Newtons/Ktex) & $24,33 \pm 18,82$ & $17,95 \pm 7,90$ & 0,5042 \\
\hline
\end{tabular}




\section{B. PRIMER PERIODO DE BALANCE}

La suplementación con Zn no tuvo efecto sobre la DAMS (Tabla 4). Sin embargo, la retención de N fue mayor en el grupo Z (Tabla 5), lo que es explicado íntegramente por el hecho de que este grupo tuvo una menor excreción de $\mathrm{N}$ por orina, ya que la digestibilidad aparente del $\mathrm{N}$ fue similar en ambos grupos.

Tabla 4: Digestibilidad aparente de la MS, primer periodo (valores expresados como promedio $\pm \mathrm{DE})$.

\begin{tabular}{|c|c|c|c|}
\hline Variable & Grupo B & Grupo Z & P \\
\hline Consumo de MS (g/d) & $353,11 \pm 3,60$ & $355,88 \pm 3,46$ & 0,249 \\
\hline Excreción de MS (g/d) & $83,06 \pm 5,81$ & $89,46 \pm 6,04$ & 0,126 \\
\hline MS Aparentemente digerida (g/d) & $270,14 \pm 4,68$ & $266,42 \pm 6,34$ & 0,323 \\
\hline Digestibilidad Aparente de la MS (\%) & $76,48 \pm 1,52$ & $74,86 \pm 1,68$ & 0,148 \\
\hline
\end{tabular}

Tabla 5: Balance de N, primer periodo (valores expresados como promedio \pm DE).

\begin{tabular}{|c|c|c|c|}
\hline Variable & Grupo B & Grupo Z & P \\
\hline Consumo de N (g/d) & $9,040 \pm 0,092$ & $9,110 \pm 0,089$ & 0,249 \\
\hline Excreción de N en heces (g/d) & $1,946+0,153$ & $2,054 \pm 0,316$ & 0,511 \\
\hline N Aparentemente digerido (g/d) & $7,094 \pm 0,198$ & $7,057 \pm 0,340$ & 0,839 \\
\hline Digestibilidad aparente del N (\%) & $78,471 \pm 1,758$ & $77,453 \pm 3,485$ & 0,576 \\
\hline Excreción de N en orina (g/d) & $3,062 \pm 0,374$ & $1,526 \pm 0,860$ & 0,006 \\
\hline Retención de N (g/d) & $4,032 \pm 0,499$ & $5,531 \pm 1,151$ & 0,028 \\
\hline Retención de N (\%) & $44,567 \pm 5,105$ & $60,692 \pm 12,471$ & 0,028 \\
\hline
\end{tabular}

\section{SEGUNDO PERIODO DE BALANCE.}

$\mathrm{Al}$ igual que en el primer periodo de balance, no hubo efecto de la suplementación con Zn sobre la DAMS (Tabla 6). La retención de $\mathrm{N}$ tendió a ser mayor en el grupo Z (Tabla 7). 
Tabla 6: Digestibilidad aparente de la MS, segundo periodo (valores expresados como promedio $\pm \mathrm{DE}$ ).

\begin{tabular}{|c|c|c|c|}
\hline Variable & Grupo B & Grupo Z & P \\
\hline Consumo de MS (g/d) & $242,36 \pm 2,52$ & $243,24 \pm 1,79$ & 0,542 \\
\hline Excreción de MS (g/d) & $60,86 \pm 11,77$ & $68,66 \pm 6,41$ & 0,230 \\
\hline MS Aparentemente digerida (g/d) & $181,50 \pm 12,23$ & $174,59 \pm 6,44$ & 0,296 \\
\hline Digestibilidad aparente de la MS (\%) & $74,88 \pm 4,86$ & $71,78 \pm 2,62$ & 0,244 \\
\hline
\end{tabular}

Tabla 7: Balance de N, segundo periodo (valores expresados como promedio \pm DE).

\begin{tabular}{|c|c|c|c|}
\hline Variable & Grupo B & Grupo Z & P \\
\hline Consumo de N (g/d) & $6,204 \pm 0,064$ & $6,227 \pm 0,046$ & 0,542 \\
\hline Excreción de N en heces (g/d) & $0,970 \pm 0,230$ & $0,917 \pm 0,152$ & 0,511 \\
\hline N Aparentemente digerido (g/d) & $5,234 \pm 0,280$ & $5,310 \pm 0,171$ & 0,621 \\
\hline Digestibilidad aparente del N (\%) & $84,34 \pm 3,85$ & $85,26 \pm 2,47$ & 0,663 \\
\hline Excreción de N en orina (g/d) & $2,083 \pm 0,557$ & $1,460 \pm 0,375$ & 0,071 \\
\hline Retención de N (g/d) & $3,151 \pm 0,643$ & $3,850 \pm 0,370$ & 0,069 \\
\hline Retención de N (\%) & $50,73 \pm 9,87$ & $61,83 \pm 6,01$ & 0,058 \\
\hline
\end{tabular}

\section{PARÁMETROS HEMATOLÓGICOS.}

Los parámetros hematológicos medidos (hematocrito, número de glóbulos rojos, concentración de hemoglobina, número de leucocitos, linfocitos y neutrófilos en sangre, (Figuras 2, 3, 4, 5, 6 y 7) no fueron modificados por la suplementación con $\mathrm{Zn}$. 


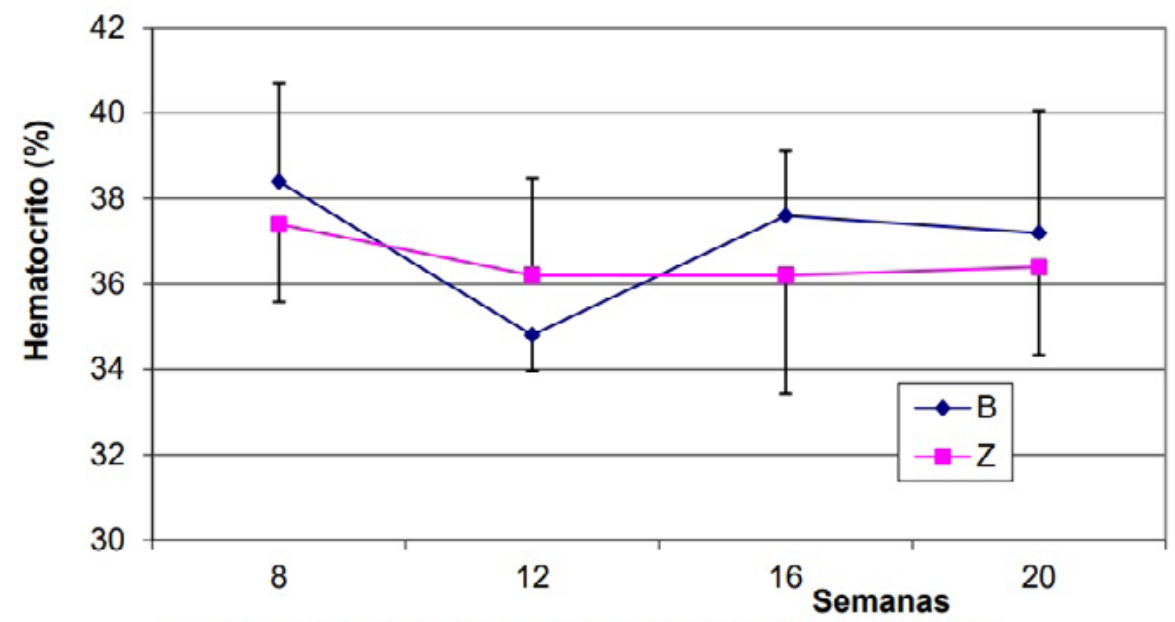

Efectos: Tratamiento: NS, Tiempo: NS, Tratamiento x Tiempo: NS.

Figura 2: Evolución del hematocrito en los corderos de ambos grupos experimentales.

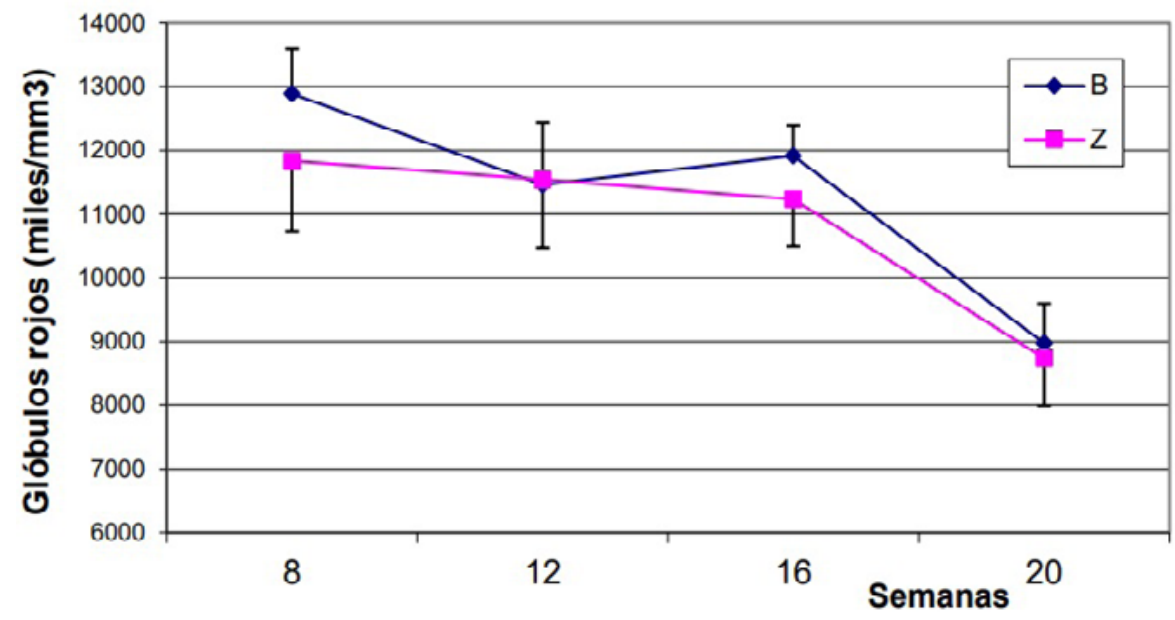

Efectos: Tratamiento: NS, Tiempo: $p<0,05$, Tratamiento x Tiempo: NS.

Figura 3: Evolución del número de glóbulos rojos en sangre de los corderos de ambos grupos experimentales. 


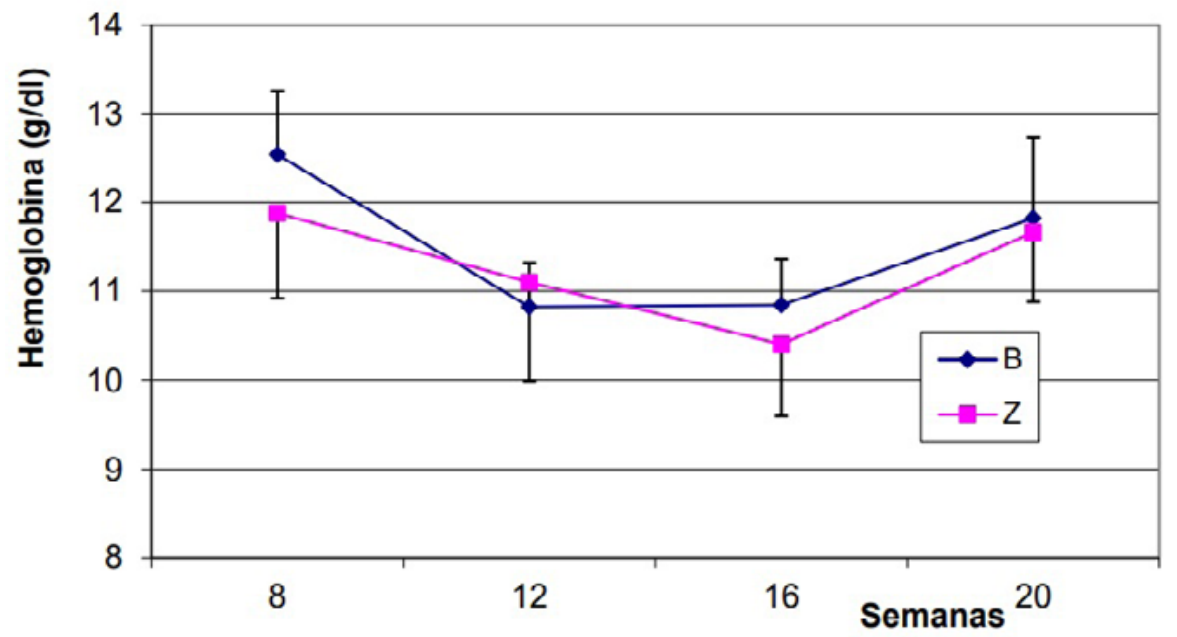

Efectos: Tratamiento: NS, Tiempo: $p<0,05$, Tratamiento $x$ Tiempo: NS.

Figura 4: Evolución de la concentración de hemoglobina en sangre en los corderos de ambos grupos experimentales.

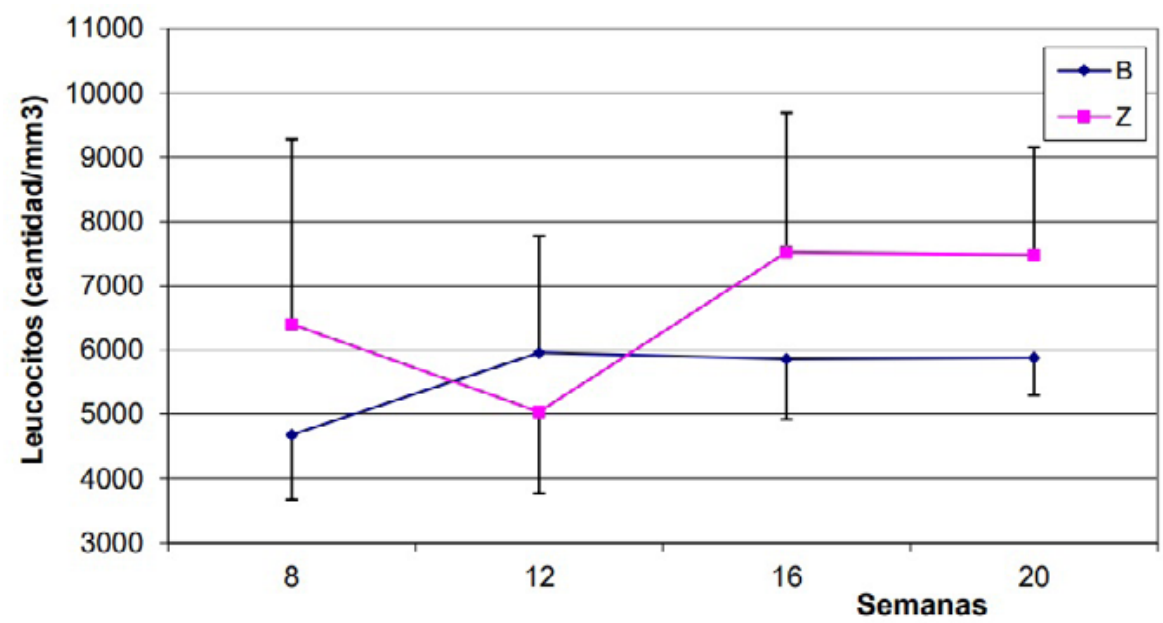

Efectos: Tratamiento: NS, Tiempo: $p<0,10$, Tratamiento $\times$ Tiempo: $p<0,05$.

Figura 5: Evolución de la concentración del número de leucocitos en sangre en los corderos de ambos grupos experimentales. 


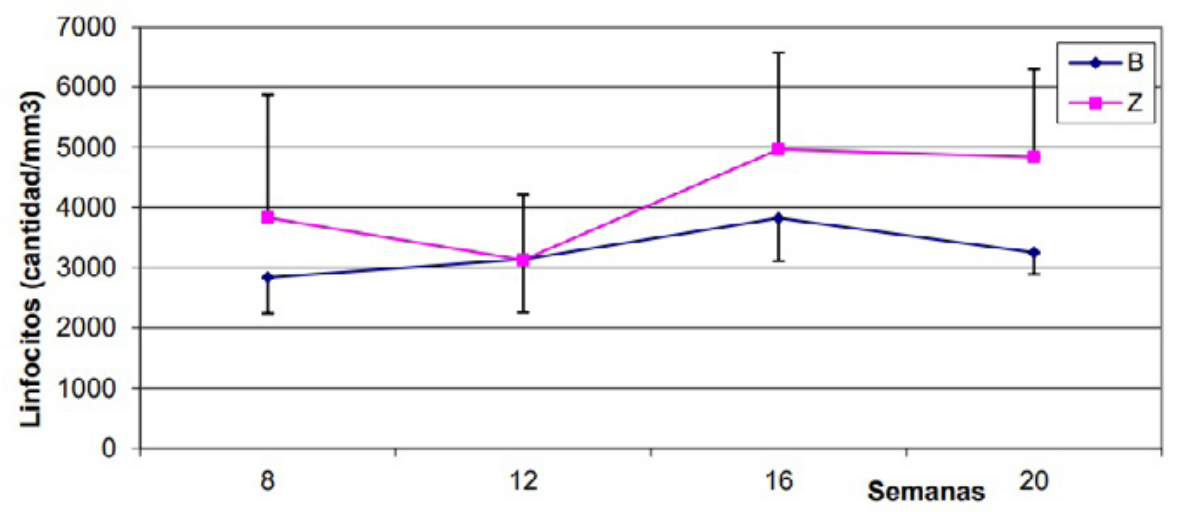

Efectos: Tratamiento: NS, Tiempo: $p<0,05$, Tratamiento $x$ Tiempo: NS.

Figura 6: Evolución del número de linfocitos en sangre en los corderos de ambos grupos experimentales.

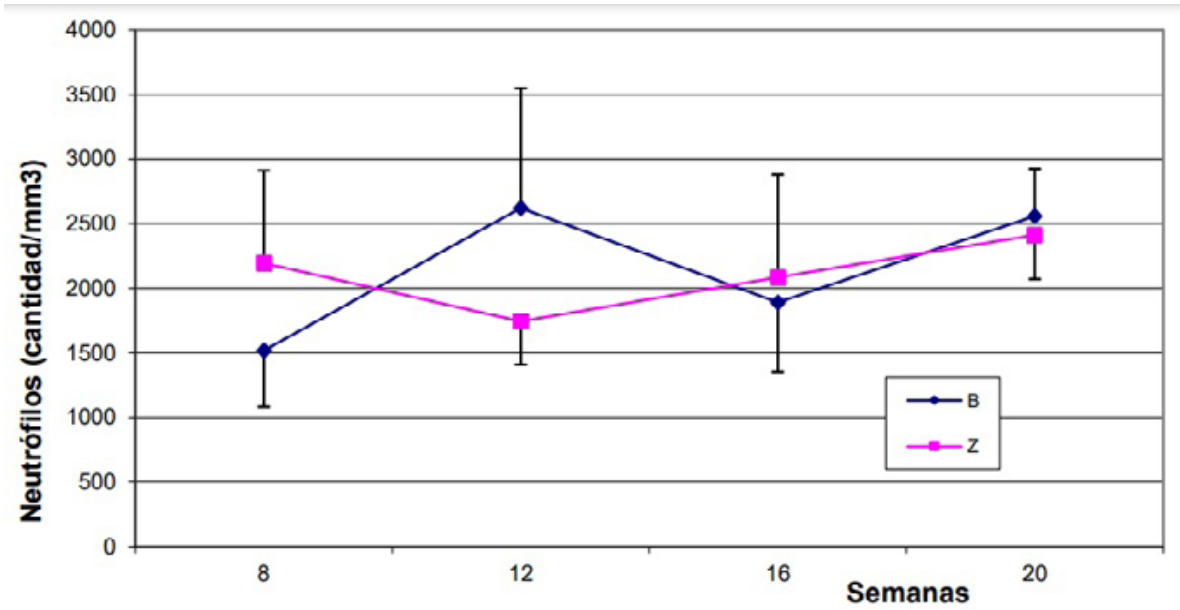

Efectos: Tratamiento: NS, Tiempo: NS, Tratamiento x Tiempo: NS.

Figura 7: Evolución del número de neutrófilos en sangre en los corderos de ambos grupos experimentales.

\section{Discusión}

White et al..$^{(9)}$, trabajando con corderos, hallaron que los grupos alimentados con 17 y 27 ppm de Zn, tenían una superior producción de lana sucia que el grupo que recibía $10 \mathrm{ppm}$ de $\mathrm{Zn}$, y también que el grupo con deficiencia clínica (con 4 ppm de Zn). En nuestro ensayo, los resultados de la suplementación son coincidentes, corroborando 
que los niveles usados son compatibles con un modelo de deficiencia subclínica de Zn, en el que está afectada significativamente la producción animal.

En el mismo sentido que nuestro estudio, White et al..$^{(9)}$ no hallaron diferencias entre las ganancias de peso de los grupos que recibían 10, 17 y 27 ppm de Zn, demostrando que éste es un parámetro menos sensible que la producción de lana, o ciertos indicadores bioquímicos (como la concentración sérica de $\mathrm{Zn}$ ), frente a la deficiencia del mineral. En un ensayo realizado con anterioridad ${ }^{(7)}$, niveles de $18 \mathrm{ppm}$ de $\mathrm{Zn}$ en la dieta experimental de los corderos no maximizaron los parámetros consumo de alimento, ganancia de peso y Zn sérico, pero las diferencias en los dos primeros parámetros fueron pequeñas con respecto al grupo inmediato superior (33 ppm de $\mathrm{Zn}$ ).

La suplementación con Zn no afectó la DAMS, lo que sugiere que 10 ppm de Zn son suficientes para el metabolismo microbiano, al menos con este tipo de dietas. Sin embargo, la adición de Zn mejoró la retención de N. Estos resultados tienen relación con el importante rol del Zn en la síntesis de proteínas y concuerdan también con los hallados por Somers y Underwood ${ }^{(11)}$, aunque estos investigadores trabajaron con un grupo deficiente con niveles dietarios más bajos (2,4 ppm de $\mathrm{Zn})$, que expresó signos clínicos de la deficiencia, y un grupo suplementado pareado por consumo. Si bien, de acuerdo a nuestro ensayo, 10 ppm de Zn no maximizaron la retención de $\mathrm{N}$, los requerimientos de Zn de corderos en crecimiento, al menos considerando esta variable, no parecen estar mucho más allá de 15 o 20 ppm, ya que no se encontró ningún efecto cuando se adicionó Zn en dietas a base de heno de festuca (Festuca arundinacea) o de pasto ovillo (Dactylis glomerata) que estaban en este rango de concentraciones ${ }^{(25)}$. En tal sentido, la suplementación con 25 ppm a partir de $\mathrm{ZnO}$ o de metionina-Zn en una dieta alta en grano de maíz que contenía 27,6 ppm de Zn, no mejoró la ganancia diaria de peso (GDP) en corderos, ni los niveles de Zn y fosfatasa alcalina en suero, así como tampoco los parámetros inmunitarios $\operatorname{medidos}^{(26)}$.

En un caso de deficiencia de $\mathrm{Zn}$ en ovinos $^{(5)}$ se halló una disminución en el hematocrito, la concentración de hemoglobina y el número de glóbulos rojos. Miller et al. ${ }^{(10)}$ también encontraron, en un ensayo con terneros Holstein, que el grupo deficiente (con severas lesiones paraqueratósicas y gran retraso del crecimiento) tenía niveles de hemoglobina menores que el grupo pareado por consumo. Otros autores también señalaron resultados similares en $\operatorname{cerdos}^{(27,28)}$, pero estos ensayos no tenían un grupo control pareado por consumo, de manera que, al menos en parte, los efectos sobre parámetros hematológicos 
pueden ser atribuidos a una disminución en el consumo de alimento. Si bien este tipo de variables puede estar más directamente relacionado con una deficiencia de hierro, también podría vincularse con una deficiencia de $\mathrm{Zn}$ debido a los efectos generales sobre la síntesis de proteínas y otros efectos metabólicos. En nuestro ensayo, luego de 5 meses de depleción, no hubo diferencias estadísticamente significativas en ninguno de los tres indicadores arriba mencionados, entre los grupos B y Z, lo que sugiere que son relativamente menos sensibles o bien requieren un tiempo mayor de depleción.

La falta de efectos sobre el conteo de leucocitos totales, linfocitos y neutrófilos con la dieta B (10 ppm de Zn) parecen coincidir con los de Droke y Spears ${ }^{(8)}$, también en corderos, que no encontraron diferencias en estos parámetros entre los grupos marginal $(8,7$ ppm de $\mathrm{Zn})$ y adecuado (43,7 ppm de $\mathrm{Zn}$ ), aunque en este caso se realizó una sola medición, a los 90 días del ensayo.

Los datos de este ensayo, conjuntamente con resultados publicados con anterioridad ${ }^{(17)}$, pueden ser interpretados bajo nuevos enfoques que han surgido en salud y nutrición humanas ${ }^{(29,30)}$. Brevemente, se trata de sumar a los biomarcadores (de tipo bioquímico, con cierta especificidad y sensibilidad, como el Zn plasmático) el aporte de los bioindicadores (de tipo funcional, no específicos, pero que responden a una suplementación con $\mathrm{Zn}$ en sujetos o poblaciones deficientes). En medicina humana, el bioindicador preferido para la deficiencia de $\mathrm{Zn}$ es el crecimiento lineal (altura) en niños y adolescentes. En ovinos, en base a nuestros resultados ${ }^{(17)}$ y a la bibliografía citada, la propuesta incluye conservar como biomarcador al $\mathrm{Zn}$ plasmático (o sérico), aunque podría considerarse también al Zn en hueso (más difícil de muestrear y relevante, probablemente, sólo en animales en crecimiento), tomando en forma conjunta la producción de lana como un bioindicador funcional, válido tanto en animales en crecimiento como en adultos.

\section{Conclusiones}

Los resultados obtenidos sugieren que la deficiencia subclínica de Zn disminuye la producción de lana en corderos, lo que puede estar relacionado con una menor retención de N. Sin embargo, al menos en las condiciones de este ensayo, no parece afectar la calidad de la lana ni la ganancia de peso en corderos, así como tampoco los parámetros hematológicos medidos. 


\section{Bibliografía}

1. Ott EA, Smith WH, Stob M, Beeson, WM. Zinc deficiency syndrome in the young lamb. J Nutr. 1964; 82(1):41-50.

2. Pierson RE. Zinc deficiency in young lambs. J Am Vet Med Assoc. 1966; 149(10):1279-82.

3. Mahmoud, OM, El Samani F, Bakheit AO, Hassan MA. Zinc deficiency in sudanese desert sheep. J Comp Path. 1983; 93(4):591-5.

4. Nelson DR, Wolff WA., Blolgett DJ, Luecke B, Ely RW, Zacchary JF. Zinc deficiency in sheep and goats: three field cases. J Am Vet Med Assoc. 1984; 184(12):1480-5.

5. Al-Saad KM, Al-Sadi HI, Abdul-Majeed MO. Clinical, hematological, biochemical and pathological studies on zinc deficiency (hypozincemia) in sheep. Vet Res. 2010; 3(2):14-20.

6. Masters DG, Chapman RE, Vaughan JD. Effects of zinc deficiency on the wool growth, skin and wool follicles of pre-ruminant lambs. Aust Journal Biol Sci. 1985; 38(4):355-64.

7. Ott EA, Smith WH, Stobb M, Parker HE, Harrington RB, Beeson WM. Zinc requirement of the growing lamb fed a purified diet. J Nutr. 1965; 87(4):459-63.

8. Droke EA, Spears JW. In vitro and in vivo immunological measurements in growing lambs fed diets deficient, marginal or adequate in zinc. J Nutr Immunol. 1993; 2(1):71-90.

9. White CL, Martin GB, Hynd PI, Chapman RE. The effect of zinc deficiency on wool growth and skin and wool follicle histology of male Merino lambs. Br J Nutr. 1994; 71(3):425-35.

10. Miller WJ, Pitts WJ, Clifton CM, Morton JD. Effects of zinc deficiency per se on feed efficiency, serum alkaline phosphatase, zinc in skin, behavior, greying, and other measurements in the Holstein calf. J Dairy Sci. 1965; 48(10):1329-34.

11. Somers M, Underwood EJ. Studies of zinc nutrition in sheep. II. The influence of zinc deficiency in ram lambs upon the digestibility of the dry matter and the utilization of the nitrogen and sulphur of the diet. Aust J Agric Res. 1969; 20(5):899-903.

12. Miller WJ, Powell GW, Hiers JM Jr. Influence of zinc deficiency on dry matter digestibility in ruminants. J Dairy Sci. 1966; 49(8):1012-3.

13. McNall AD, Etherton TD, Fosmire GJ. The impaired growth induced by zinc deficiency in rats is associated with decreased expression of the hepatic insulin-like growth factor I and growth hormone receptor genes. J Nutr. 1995; 125(4):874-9.

14. Droke EA, Spears JW, Armstrong JD, Kegley EB, Simpson RB. Dietary zinc affects serum concentration on insulin and insulin-like growth factor I in growing lambs. J Nutr. 1993; 123(1):13-19.

15. MacDonald RS. The role of zinc in growth and cell proliferation. J Nutr. 2000; 130(5):1500S-8S.

16. Fraker PJ, King LE. Reprogramming of the immune system during zinc deficiency. Annu Rev Nutr. 2004; 24(1):277-98.

133 Efecto de la deficiencia subclínica de zinc en corderos sobre retención de nitrógeno, parámetros productivos y hematológicos 
17. Pechin GH, Corbellini CN, Cseh SB, Stritzler NP. Efecto de la deficiencia subclínica de zinc en corderos sobre parámetros bioquímicos, concentración tisular y balance de zinc. Invet. 2018; 20(1-2):243-54.

18. National Research Council. Nutrient Requirements of Small Ruminants. Washington, D.C., USA: National Academies Press; 2007.

19. International Wool Textile Organization (IWTO). Test Method Number IWTO-12-00: Measurement of the mean and distribution of fibre diameter using the Sirolan Laserscan fibre diameter analyzer. Ilkley, U.K.: Woolmark Co; 2001.

20. Dabbs TP, van Schie H, Glass M. The effect of fibre curvature on Laserscan diameter measurement. IWTO Technology and Standards Committee Meeting. Nice, France. December 1994. Report 2.

21. International Wool Textile Organization (IWTO). Test Method Number IWTO-30-98: Determination of staple length and staple strength. Ilkley, UK: Woolmark Co; 2002.

22. Association of Official Analytical Chemists (AOAC). Official Methods of Analysis. $15^{\text {th }}$ Ed. Arlington, Virginia, USA: AOAC; 1990.

23. Littell RC, Henry PR, Ammermann CB. Statistical analysis of repeated measures data using SAS procedures. J Anim Sci. 1998; 76(4):1216-31.

24. SAS Institute Inc. SAS/STAT 9.1 User's Guide. Cary, NC, USA. 2004.

25. Kegley EB, Spears JW. Effect of zinc supplementation on performance and zinc metabolism of lambs fed forage-based diets. J Agric Sci (Cambridge). 1994; 123(2):287-92.

26. Droke EA, Gengelbach GP, Spears JW. Influence of level and source (inorganic vs organic) of zinc supplementation on immune function in growing lambs. Asian-australas J Anim Sci. 1998; 11(2):139-44.

27. Hoefer JA, Miller ER, Ullrey DE, Ritche HD, Luecke RW. Interrelationships between calcium, zinc, iron and copper in swine feeding. J Anim Sci. 1960; 19(1):249-259.

28. Miller ER, Luecke RW, Ullrey DE, Blaltzer BV, Bradley BL, Hoefer JA. Biochemical, skeletal and allometric changes due to zinc deficiency in the baby pig. J Nutr. 1968; 95(2): 278-286.

29. Raiten DJ, Combs GF Jr. Directions in nutritional assessment. Shigt and life. 2015; 29 (1): 39-44.

30. King JC, Brown KH, Gibson RS, Krebs NF, Lowe NM, Siekman JH, Raiten DJ. Biomarkers of nutrition for development (BOND) - Zinc Review. J Nutr. 2016; 146(Suppl.): 858S-85S. 